RASĀYAN J. Chem.

Vol. 12 | No. 4 |2260 - 2266| October - December | 2019 ISSN: 0974-1496 | e-ISSN: 0976-0083 | CODEN: RJCABP

\title{
SYNTHESIS AND ANTIMICROBIAL ACTIVITY OF NOVEL 4- AMINO BENZAMIDE DERIVED 1,2,3 - TRIAZOLE LINKED PYRAZOLINES
}

\author{
S. Sathiya ${ }^{1}$, S. Nandhabala ${ }^{1}$, N. Hari ${ }^{2}$, R. Paranthaman ${ }^{3}$, A. Sankar ${ }^{4}$ \\ and R. Ravikumar ${ }^{1, *}$ \\ ${ }^{1}$ Chemistry Department, Vivekanandha College of Arts and Sciences for Women, \\ Tiruchengode-637205, Tamilnadu, India \\ ${ }^{2}$ NMR Laboratory, School of Chemical and Biotechnology, SASTRA Deemed University, \\ Thirumalaisamudram-613401, Thanjavur, India \\ ${ }^{3}$ Microbiology Department, Vivekanandha College of Arts and Sciences for Women, \\ Tiruchengode-637205, Tamilnadu, India \\ ${ }^{4}$ Chemistry Department, Kandaswami Kandar's College, Namakkal-638182, Tamilnadu, India \\ *E-mail: drravikumarvicas@gmail.com
}

\begin{abstract}
In the view of investigating the antimicrobial potential of novel 4- amino benzamide derived 1, 2, 3 triazole-linked chalcones and their pyrazolines derivatives, the present work was designed and synthesized as given in the scheme mentioned. All the compounds were characterized by the physicochemical methods and all the compounds were subjected to in vitro antimicrobial studies.

Keywords: 1, 2, 3- Triazole, Chalcone, Pyrazoline, FTIR, ${ }^{1} \mathrm{H}$ NMR, ${ }^{13} \mathrm{C}$ NMR, Mass Spectra and Antimicrobial Activity.
\end{abstract}

(C) RASĀYAN. All rights reserved

\section{INTRODUCTION}

There is a need to focus on developing new molecules to treat infectious diseases due to the resistance acquired by the microbes towards the chemical compounds on prolonging the interaction. ${ }^{1,2}$ Binding capacity of 1,2,3-triazole moiety ${ }^{3}$ towards the biological targets is attributed to its physical and chemical features like high aromatic stability and dipole moment ${ }^{4}$. We designed a molecule containing both the important heterocycles namely triazole and pyrazoline and to compare the microbial activity of the 1,2,3triazole linked chalcone and their pyrazoline derivatives. The previous results ${ }^{5}$ on the above-mentioned chalcones prompted us to carry out the present work. Chalcones and its derivatives have attracted the attention of the chemists owing to their many fold pharmacological activities like antimalarial, anticancer, antiprotozoal, anti-inflammatory, antifungal ${ }^{6-10}$, larvicidal, anticonvulsant, antioxidant, enzyme inhibition and antimitotic activity ${ }^{11}$ Similar to triazoles the chalcones and the pyrazoline derivatives also reported to possess diverse biological activities. Many numbers of different heterocyclic moieties containing organic compounds like azetidinones ${ }^{12}$, benzathiazole ${ }^{13}$, quinazoline ${ }^{14}$,pyrazole -3 -carboxamide ${ }^{15}$ have been studied for their anti-microbial activity. The anti-microbial nature of an organic compound is not specific to any chemical moieties or elements was concluded from the results of those studies. Hence, in the present work, the title compounds were subjected to microbial activity and the results are presented in Tables 1 and 2. Pyrazolines linked heterocyclic compounds constitute an important class of compound for new drug development due to their diverse pharmacological activities such as antimicrobial activity ${ }^{16,17}$, anticancer ${ }^{18}$, antihyperglycemic activity ${ }^{19}$, antimalarial activity ${ }^{20}$, antidepressant and anticonvulsant ${ }^{21}$, MAO inhibitors ${ }^{22,23}$, antitubercular activity ${ }^{24,25}$, Cannabinoid receptor antagonist activity ${ }^{26}$, COX-2 inhibitor activity ${ }^{27}$, antihepatotoxic activity. ${ }^{28}$ The present study is to ensure the microbial activity of

Rasayan J. Chem., 12(4), 2260-2266(2019)

http://dx.doi.org/10.31788/RJC.2019.1245383

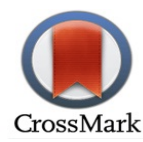


RASĀYAN J. Chem.

Vol. 12 | No. 4 |2260 - 2266| October - December | 2019

chalcones studied in our previous work and also to evaluate the biological activity of 1,2,3-triazole linked pyrazolines. We report the synthesis and antimicrobial activity of 4-aminobenzamide derived chalcones and their pyrazoline derivatives (Scheme-1).

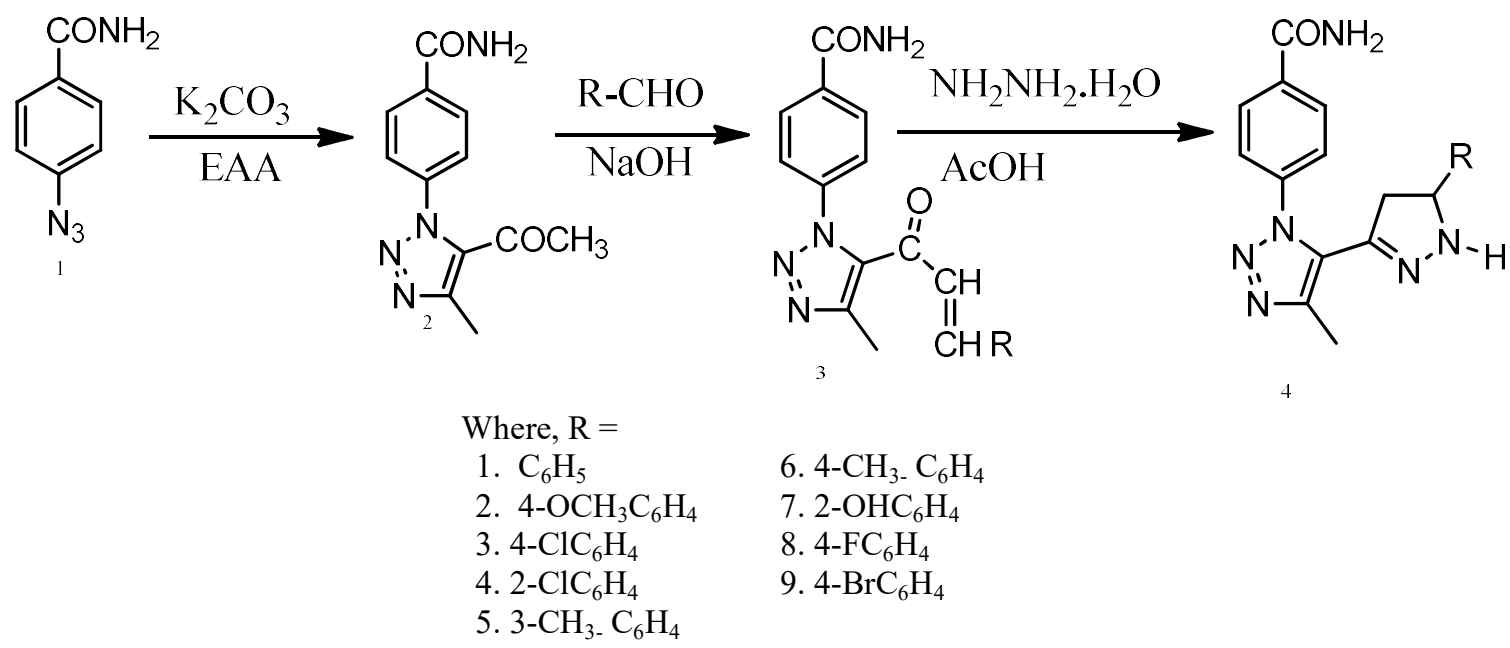

Scheme- 1: Synthesis of Pyrazoline Derivative

\section{Materials and Methods}

\section{EXPERIMENTAL}

Solvents and chemicals purchased from Merck were used. The completion of the reaction was ensured using pre-coated Aluminium TLC plates. The compounds were purified by conventional column chromatography and preparative TLC techniques. Melting points were measured by open capillaries in a sulphuric acid bath method and are uncorrected. IR, ${ }^{1} \mathrm{H}$ NMR ${ }^{13} \mathrm{C}$ NMR and mass spectra were recorded on Perkin-Elmer model 1620 FTIR spectrophotometer, Brucker AvanceII $300 \mathrm{MHz}$ spectrophotometer and JEOL mass spectrometer respectively.

\section{Antimicrobial Activity}

Microbial cultures obtained from the IMTECH, Chandigarh, India were used for the evaluation of antimicrobial activity following the disc diffusion method ${ }^{29}$. The test organisms used were Bacillus subtilis, Bacillus cereus, Staphylococcus aureus, Escherichia coli, Micrococcus, Pseudomonas fluorescens, Pseudomonas aeruginosa, Enterococcus faecalis, Klebsiella and Salmonella typhi, Lactobacillus. Standard drug ciprofloxacin was used as a reference. The zone of inhibition was expressed in $\mathrm{mm}$ and the result is presented in Table- 1 and 2.

\section{Reaction Procedure for the Synthesis of p-Azido Benzamide ${ }^{30}$}

p-aminobenzamide $(0.136 \mathrm{~g}, 1 \mathrm{mmol})$ was dissolved in $50 \mathrm{ml}$ of $1: 1 \mathrm{HCl}$ is taken in a round bottom flask, equimolar quantity of sodium nitrite $(0.069 \mathrm{~g}, 1 \mathrm{mmol}),(0.065 \mathrm{~g}, 1 \mathrm{mmol})$ sodium azide dissolved in $25 \mathrm{ml}$ of ice-cold water was added with stirring in an ice bath. The yield was $98 \%$.

\section{Spectral Data of the Compound}

p- Azido Benzamide (Colourless amorphous powder) (1): $\mathrm{MP} 95^{\circ} \mathrm{C}$; $\mathrm{IR}\left(\mathrm{KBr}, \mathrm{cm}^{-1}\right): 3450,3120,1690$, 1513; ${ }^{1} \mathrm{H}$ NMR (DMSO-d $\left.6,300 \mathrm{MHz}\right): 7.93(2 \mathrm{H}, \mathrm{d}, \mathrm{J}=1.8 \mathrm{~Hz}, \mathrm{Ar}), 7.20(2 \mathrm{H}, \mathrm{d}, \mathrm{J}=1.8 \mathrm{~Hz}, \mathrm{Ar}), 7.38(2 \mathrm{H}, \mathrm{s}$, $\left.\mathrm{CONH}_{2}\right) ;{ }^{13} \mathrm{C}$ NMR (DMSO-d 6 , $300 \mathrm{MHz}$ ): 167.46, 167.06, 144.38, 142.76, 131.68, 129.87, 119.57; MS: $m / z 162\left[\mathrm{M}^{+}\right]$.

\section{Procedure for the Synthesis of 4-(5-acetyl-4-methyl-1H-1, 2, 3-triazole-1-yl) Benzamide ${ }^{31}$}

p-azido benzamide $(0.162 \mathrm{~g}, 1 \mathrm{mmol})$ was mixed with acetylacetone $(1 \mathrm{~g}, 1 \mathrm{mmol})$ and $\mathrm{K}_{2} \mathrm{CO}_{3}(0.414 \mathrm{~g}, 3$ $\mathrm{mmol})$ in $20 \mathrm{ml}$ of $\left(95 \%\right.$ ethanol) were mixed and the mixture was heated for $6 \mathrm{~h}$ at $80^{\circ} \mathrm{C}$. The yield was 94 $\%$. 
RASĀYAN J. Chem.

Vol. 12 | No. 4 |2260 - 2266| October - December | 2019

Table-1: Antibacterial Activity of 1, 2,3,-Triazole-linked Chalcone Using Streptomycin As A Standard Drug-Zone of Inhibition in $\mathrm{mm}$

\begin{tabular}{c|c|c|c|c|c|c|c|c|c|c}
\hline Micro organism & 3.1 & 3.2 & 3.3 & 3.4 & 3.5 & 3.6 & 3.7 & 3.8 & 3.9 & Std. Drug \\
\hline Bacillus cereus & - & 14 & 12 & 13 & - & - & 16 & 17 & 18 & 25 \\
\hline Bacillus subtilis & 08 & 13 & 12 & 11 & - & 12 & - & 11 & 12 & 20 \\
\hline Escherichia coli & - & 11 & 14 & 16 & - & 14 & 13 & 13 & 12 & 18 \\
\hline Enterococcus faecalis & 19 & 12 & 14 & 17 & 13 & - & 21 & 16 & 18 & 25 \\
\hline Klebseilla & & - & 20 & 16 & - & - & 12 & 16 & 20 & 24 \\
\hline Lactobacillus & & - & 21 & 19 & & 13 & & - & 20 & 28 \\
\hline Micrococcus & 13 & - & 08 & 07 & 17 & - & 0.8 & 17 & 18 & 26 \\
\hline Pseudomonas fluorescens & 06 & 06 & 12 & 12 & - & 12 & 12 & 11 & 11 & 20 \\
\hline Pseudomonas aeruginosa & 05 & 05 & 11 & 15 & - & - & 14 & 20 & 18 & 26 \\
\hline Staphylococcus aureus & 07 & 12 & 12 & 14 & - & 11 & 12 & 12 & 13 & 20 \\
\hline Salmonella typhi & 03 & 13 & 15 & 14 & - & 12 & 14 & 12 & 13 & 19 \\
\hline
\end{tabular}

Table-2: Antibacterial Activity of 1, 2,3-Triazole-linked Pyrazoline Using Streptomycin As A Standard Drug- zone of Inhibition in $\mathrm{mm}$

\begin{tabular}{c|c|c|c|c|c|c|c|c|c|c}
\hline Microorganism & 4.1 & 4.2 & 4.3 & 4.4 & 4.5 & 4.6 & 4.7 & 4.8 & 4.9 & $\begin{array}{c}\text { Std. } \\
\text { Drug }\end{array}$ \\
\hline Bacillus cereus & - & - & 14 & 17 & 02 & - & 14 & - & 20 & 28 \\
\hline Bacillus subtilis & - & 07 & 15 & 13 & 04 & 19 & - & 17 & 11 & 20 \\
\hline Escherichia coli & - & - & 12 & 16 & - & 14 & 12 & 13 & 12 & 21 \\
\hline Enterococcus faecalis & 19 & - & 16 & 15 & - & 16 & 15 & 17 & 16 & 25 \\
\hline Klebseilla & - & 04 & 16 & 10 & - & 17 & 11 & 16 & 20 & 28 \\
\hline Lactobacillus & & & 17 & 12 & - & 13 & 12 & - & 20 & 25 \\
\hline Micrococcus & 13 & - & & 09 & - & 14 & 0.8 & 15 & 16 & 18 \\
\hline $\begin{array}{c}\text { Pseudomonas } \\
\text { fluorescens }\end{array}$ & - & 06 & 15 & 13 & 05 & 12 & 12 & 11 & 11 & 20 \\
\hline $\begin{array}{c}\text { Pseudomonas } \\
\text { aeruginosa }\end{array}$ & - & 08 & 08 & 09 & 12 & - & 08 & 20 & 18 & 21 \\
\hline $\begin{array}{c}\text { Staphylococcus } \\
\text { aureus }\end{array}$ & 10 & - & 09 & 07 & - & 11 & 11 & 12 & 13 & 18 \\
\hline \begin{tabular}{c} 
Salmonella typhi \\
\hline
\end{tabular} & - & - & 10 & 09 & - & 12 & 14 & 12 & 13 & 16 \\
\hline
\end{tabular}

\section{Spectral Data of the Compound}

4-(5-Acetyl-4-Methyl-1H-1, 2, 3-Triazole-1-YI)Benzamide (Colourless Amorphous Powder) (2): MP $118^{\circ} \mathrm{C}$; IR $\left(\mathrm{KBr}, \mathrm{cm}^{-1}\right): 3457,3125,1697,1570,1511,1310 ;{ }^{1} \mathrm{H}$ NMR (DMSO-d $\left.6300 \mathrm{MHz}\right): 7.76(2 \mathrm{H}, \mathrm{d}$, $\mathrm{J}=8.4 \mathrm{~Hz}, \mathrm{Ar}), 8.13(2 \mathrm{H}, \mathrm{d}, \mathrm{J}=8.7 \mathrm{~Hz}, \mathrm{Ar}), 2.64\left(3 \mathrm{H}, \mathrm{s}, \mathrm{COCH}_{3}\right), 2.55\left(3 \mathrm{H}, \mathrm{s}, \mathrm{CH}_{3}\right), 7.62,\left(2 \mathrm{H}, \mathrm{s}, \mathrm{CONH}_{2}\right)$; ${ }^{13} \mathrm{C}$ NMR (DMSO-d $\left.6,300 \mathrm{MHz}\right): 193.77,167.24,143.39,138.24,137.50,136.03,129.35,125.57,28.05$, 10.20; MS: m/z $244.1\left[\mathrm{M}^{+}\right]$.

General Reaction Procedure for 4-(4-Methyl-5-(3-Phenylacryloyl)-1H-1,2,3-Triazole-1YI)Benzamide ${ }^{31}(3.1-3.9)$.

4-(5-acetyl-4-methyl-1H-1, 2, 3-triazole-1-yl)benzamide $(0.332 \mathrm{~g}, 1 \mathrm{~m} \mathrm{~mol})$ and aromatic aldehydes in the ratio of (1: 1. 5) were mixed with $10 \mathrm{~mL}$ of ethanol solvent and $20 \mathrm{~mL}$ of $25 \% \mathrm{NaOH}$ catalyst in a round bottom flask were stirred for $5 \mathrm{~h}$ at room temperature. The yield was $98 \%$.

The characterization of chalcones numbered (3.2- 3.9 except 3.1 and 3.7) were established in our previous paper $^{5}$. Characterisation of the chalcone not reported in our previous work namely 4-(5-(3-(2hydroxyphenyl) acryloyl)-4-methyl-1H-1,2,3-triazole-1-yl)benzamide and 4-(4-methyl-5-(3phenylacryloyl)-1H-1,2,3-triazole-1-yl)benzamide are reported here. 
Spectral Data of the Compounds (3.1 and 3.7)

4-(4-Methyl-5-(3-Phenylacryloyl)-1H-1,2,3-Triazol-1-Yl)Benzamide (Pale Yellow Coloured Solid) (3.1):MP $234^{\circ} \mathrm{C}$; IR $\left(\mathrm{KBr}, \mathrm{cm}^{-1}\right)$ : 3455, 3110, 1698, 1512, 13210; ${ }^{1} \mathrm{HNMR}$ (DMSO-d6, $300 \mathrm{MHz}$ ): 7.80 $(2 \mathrm{H}, \mathrm{d}, \mathrm{J}=8.4 \mathrm{~Hz} \mathrm{Ar}), 8.4(2 \mathrm{H}, \mathrm{d}, \mathrm{J}=8.4 \mathrm{~Hz}, \mathrm{Ar}), 8.07(1 \mathrm{H}, \mathrm{d}, \mathrm{H \alpha}, \mathrm{J}=16.2 \mathrm{~Hz}), 8.14\left(1 \mathrm{H}, \mathrm{d}, \mathrm{H}_{\beta}, \mathrm{J}=18.4 \mathrm{~Hz}\right)$, 7.5-7.9 (5H, m, Ar), $2.65\left(3 \mathrm{H}, \mathrm{s}, \mathrm{CH}_{3}\right), 7.63\left(2 \mathrm{H}, \mathrm{s}, \mathrm{CONH}_{2}\right) ;{ }^{13} \mathrm{C}$ NMR (DMSO-d6, $\left.300 \mathrm{MHz}\right): 183.76$, 167.25 , 143.64, 139.56, 137.54, 136.10, 134.82, 131.28, 129.55, 129.37, 129.18, 125.65, 123.16, 10.45, EI-MS m/z: $332.36\left[\mathrm{M}^{+}\right]$.

4-(5-(3-(2-Hydroxyphenyl) Acryloyl)-4-Methyl-1H-1,2,3-Triazol-1-Yl)Benzamide (Pale Yellow Coloured Solid) (3.7): MP $247^{\circ} \mathrm{C}$; IR $\left(\mathrm{KBr}, \mathrm{cm}^{-1}\right): 3456,3100,1695,1550,1290 ;{ }^{1} \mathrm{HNMR}$ (DMSO-d6, $300 \mathrm{MHz}): 8.11(2 \mathrm{H}, \mathrm{d}, \mathrm{J}=6.0 \mathrm{~Hz} \mathrm{Ar}), 7.80(2 \mathrm{H}, \mathrm{d}, \mathrm{J}=8.4 \mathrm{~Hz}, \mathrm{Ar}), 8.21(1 \mathrm{H}, \mathrm{d}, \mathrm{H \alpha}, \mathrm{J}=24.3 \mathrm{~Hz}), 7.71(1 \mathrm{H}$, $\left.\mathrm{d}, \mathrm{H}_{\beta}, \mathrm{J}=24.6 \mathrm{~Hz}\right), 7.32(1 \mathrm{H}, \mathrm{t}, \mathrm{J}=7.8 \mathrm{~Hz}, \operatorname{Ar}), 6.92(1 \mathrm{H}, \mathrm{t}, \mathrm{J}=7.5 \mathrm{~Hz}, \operatorname{Ar}), 6.98(1 \mathrm{H}, \mathrm{d}, \mathrm{J}=9.1 \mathrm{~Hz}, \operatorname{Ar}), 8.14$ $(1 \mathrm{H}, \mathrm{d}, \mathrm{J}=9.1 \mathrm{~Hz}, \mathrm{Ar}), 2.64\left(3 \mathrm{H}, \mathrm{s}, \mathrm{CH}_{3}\right), 10.43\left(2 \mathrm{H}, \mathrm{s}, \mathrm{CONH}_{2}\right) ;{ }^{13} \mathrm{C}$ NMR (DMSO-d6, $300 \mathrm{MHz}$ ): $184.26,167.26,158.02,143.82,139.60,139.34,137.61,136.06,132.60,129.83,129.35,125.69,122.66$, 121.64, 120.04, 116.81, 10.44; EI-MS m/z: $348\left[\mathrm{M}^{+}\right]$.

General Reaction Procedure for the Synthesis of 4-(4-Methyl-5-(5-Phenyl-4,5-Dihydro-1H-Pyrazol3-YI)-1H-1,2,3-Triazol-1-YI)Benzamide, 4.1-4.9.

4-(4-methyl-5-(3-phenylacryloyl)-1H-1,2,3-triazol-1-yl)benzamide $(0.332 \mathrm{~g}, 1 \mathrm{mmol})$ was mixed with hydrazine hydrate $(0.75 \mathrm{~g}, 1.5 \mathrm{mmol})$ in a round bottom flask. To this $20 \mathrm{ml}$ of ethanol and $5 \mathrm{ml}$ of $20 \%$ glacial acetic acid were added and heated with stirring for $8 \mathrm{~h}$ at $80^{\circ} \mathrm{C}$. Yield obtained was $85 \%$.

4-(4-Methyl-5-(5-Phenyl-4,5-Dihydro-1H-Pyrazol-3-YI)-1H-1,2,3-Triazol-1-Yl)Benzamide (Colourless Amorphous Solid) (4.1): MP $220^{\circ} \mathrm{C}$; IR (KBr, cm $\left.{ }^{-1}\right): 3315,3010,1695,1525,1343 ;{ }^{1} \mathrm{H}$ NMR (DMSO-d $6,300 \mathrm{MHz}): 8.06(2 \mathrm{H}, \mathrm{d}, \mathrm{J}=8.1 \mathrm{~Hz}, \mathrm{Ar}), 7.76(2 \mathrm{H}, \mathrm{d}, \mathrm{J}=8.4 \mathrm{~Hz}, \mathrm{Ar}), 7.2-7.42(5 \mathrm{H}, \mathrm{m}, \mathrm{Ar})$, $3.65\left(1 \mathrm{H}, \mathrm{dd}, \mathrm{J}=10.8 \mathrm{~Hz}, \mathrm{CH}_{2}\right), 2.97(1 \mathrm{H}, \mathrm{dd}, \mathrm{J}=10.2 \mathrm{~Hz} \mathrm{CH})_{2}, 4.81(1 \mathrm{H}, \mathrm{t}, \mathrm{J}=2.7 \mathrm{~Hz}, \mathrm{C}-\mathrm{H}), 4.60(1 \mathrm{H}, \mathrm{s}, \mathrm{N}-$ $\mathrm{H}), 10.0\left(\mathrm{~s}, \mathrm{CONH}_{2}\right), 2.57\left(3 \mathrm{H}, \mathrm{s}, \mathrm{CH}_{3}\right) ;{ }^{13} \mathrm{C} \mathrm{NMR}\left(\mathrm{DMSO}_{\mathrm{d}}, 300 \mathrm{MHz}\right) \delta: 165.2,143.87,143.31$, 139.64, 138.08, 134.64, 132.20, 128.90, 128.79, 127.63, 127.07, 125.35, 63.08, 42.44, 10.47; MS: $\mathrm{m} / \mathrm{z}$ $346.39\left[\mathrm{M}^{+}\right]$.

4-(5-(5-(4-Methoxyphenyl)-4,5-Dihydro-1H-Pyrazol-3-Yl)-4-Methyl-1H-1,2,3-Triazol-1Yl)Benzamide (Colourless Amorphous Solid) (4.2)

MP 239 ${ }^{\circ}$; IR (KBr, cm $\left.{ }^{-1}\right): 3327,3089,1610,1519,1342 ;{ }^{1} \mathrm{H}$ NMR (DMSO-d $\left.6,300 \mathrm{MHz}\right): 7.76$ (2H, d, $\mathrm{J}=8.4 \mathrm{~Hz}, \mathrm{Ar}), 8.06(2 \mathrm{H}, \mathrm{d}, \mathrm{J}=7.8 \mathrm{~Hz}, \mathrm{Ar}), 6.93(2 \mathrm{H}, \mathrm{d}, \mathrm{J}=8.4 \mathrm{~Hz}, \mathrm{Ar}), 7.33(2 \mathrm{H}, \mathrm{d}, \mathrm{J}=8.4 \mathrm{~Hz}, \mathrm{Ar}), 3.0(1 \mathrm{H}$, $\left.\mathrm{dd}, \mathrm{J}=10.2 \mathrm{~Hz}, \mathrm{CH}_{2}\right), 3.59\left(1 \mathrm{H}, \mathrm{dd}, \mathrm{J}=10.2 \mathrm{~Hz}, \mathrm{CH}_{2}\right), 4.82(1 \mathrm{H}, \mathrm{t}, \mathrm{J}=2.7 \mathrm{~Hz}, \mathrm{C}-\mathrm{H}), 4.59(1 \mathrm{H}, \mathrm{s}, \mathrm{N}-\mathrm{H}), 10.0$ $\left(2 \mathrm{H}, \mathrm{s}, \mathrm{CONH}_{2}\right), 2.56\left(3 \mathrm{H}, \mathrm{s}, \mathrm{CH}_{3}\right), 3.74\left(3 \mathrm{H}, \mathrm{s}, \mathrm{OCH}_{3}\right) ;{ }^{13} \mathrm{C}$ NMR (DMSO-d $\left.6.300 \mathrm{MHz}\right): 165.22,158.93$, $143.94,139.70,138.09,135.14,134.63,132.13,128.79,128.19,125.34,114.27,62.63,55.54,42.39$, 10.47; MS: $m / z 376.41\left[\mathrm{M}^{+}\right]$.

4-(5-(5-(4-Chlorophenyl)-4,5-Dihydro-1H-Pyrazol-3-Yl)-4-Methyl-1H-1,2,3-Triazol-1-

Yl)Benzamide (Colourless Amorphous Solid) (4.3): MP 244 ${ }^{\circ}$; IR (KBr, $\left.\mathrm{cm}^{-1}\right): 3325,3078,1599$, 1523, 1345; ${ }^{1} \mathrm{H}$ NMR (DMSO-d $\left.\mathrm{d}_{6} 300 \mathrm{MHz}\right): 8.06(2 \mathrm{H}, \mathrm{d}, \mathrm{J}=8.4 \mathrm{~Hz}, \mathrm{Ar}), 7.76(2 \mathrm{H}, \mathrm{d}, \mathrm{J}=8.7 \mathrm{~Hz}, \mathrm{Ar}), 7.43$ $(4 \mathrm{H}, \mathrm{s}, \mathrm{Ar}), 3.66\left(1 \mathrm{H}, \mathrm{dd}, \mathrm{J}=10.8 \mathrm{~Hz}, \mathrm{CH}_{2}\right), 3.00\left(1 \mathrm{H}, \mathrm{dd}, \mathrm{J}=10.2 \mathrm{~Hz}, \mathrm{CH}_{2}\right), 4.81(1 \mathrm{H}, \mathrm{t}, \mathrm{J}=3.0 \mathrm{~Hz}, \mathrm{CH})$, $4.62(1 \mathrm{H}, \mathrm{s}, \mathrm{NH}), 10.0\left(2 \mathrm{H}, \mathrm{s}, \mathrm{CONH}_{2}\right), 2.56\left(3 \mathrm{H}, \mathrm{s}, \mathrm{CH}_{3}\right) ;{ }^{13} \mathrm{C}$ NMR (DMSO-d 6 , $\left.300 \mathrm{MHz}\right): 165.20$, $143.98,142.38,139.53,138.05,134.65,132.27,132.11,129.00,128.85,128.79,125.35,62.32,42.40$, 10.46; MS: $m / z 380.83\left[\mathrm{M}^{+}\right], 382.83[\mathrm{M}+2]$.

4-(5-(5-(2-Chlorophenyl)-4,5-Dihydro-1H-Pyrazol-3-Yl)-4-Methyl-1H-1,2,3-Triazol-1-

Yl)Benzamide (Colourless Amorphous Solid) (4.4): MP 241 ${ }^{\circ} \mathrm{C}$; IR $\left(\mathrm{KBr}, \mathrm{cm}^{-1}\right): 3359,2928,1609$, 1522, 1346; ${ }^{1} \mathrm{H}$ NMR (DMSO-d $\left.\mathrm{D}_{6}, 300 \mathrm{MHz}\right): 8.06(2 \mathrm{H}, \mathrm{d}, \mathrm{J}=8.1 \mathrm{~Hz}, \mathrm{Ar}), 7.76(2 \mathrm{H}, \mathrm{d}, \mathrm{J}=7.2 \mathrm{~Hz}, \mathrm{Ar})$, $7.59(1 \mathrm{H}, \mathrm{d}, \mathrm{J}=7.2 \mathrm{~Hz}, \mathrm{Ar}), 7.51(1 \mathrm{H}, \mathrm{d}, \mathrm{J}=7.5 \mathrm{~Hz}, \operatorname{Ar}), 7.37(2 \mathrm{H}, \mathrm{t}, \mathrm{J}=7.5 \mathrm{~Hz}, \mathrm{Ar}), 3.78(1 \mathrm{H}, \mathrm{dd}, \mathrm{J}=11.4 \mathrm{~Hz}$, $\left.\mathrm{CH}_{2}\right), 2.93\left(1 \mathrm{H}, \mathrm{dd}, \mathrm{J}=9.9 \mathrm{~Hz}, \mathrm{CH}_{2}\right), 5.13(1 \mathrm{H}, \mathrm{t}, \mathrm{J}=8.7 \mathrm{~Hz}, \mathrm{CH}), 4.65(1 \mathrm{H}, \mathrm{s}, \mathrm{NH}), 10.01\left(2 \mathrm{H}, \mathrm{s}, \mathrm{CONH}_{2}\right)$, 
$2.57\left(3 \mathrm{H}, \mathrm{s}, \mathrm{CH}_{3}\right) ;{ }^{13} \mathrm{C}$ NMR (DMSO-d $\left.6,300 \mathrm{MHz}\right): 165.19,143.62,140.56,139.44,138.03,134.66$, $132.34,132.30,129.91,129.35,128.78,128.12,127.91,125.36,59.94,41.43,10.46$; MS: $\mathrm{m} / \mathrm{z} 380.83$ $\left[\mathrm{M}^{+}\right], 382.83[\mathrm{M}+2]$.

\section{4-(4-Methyl-5-(5-(M-Tolyl)-4,5-Dihydro-1H-Pyrazol-3-Yl)-1H-1,2,3-Triazol-1-Yl)Benzamide}

(Colourless Amorphous Solid) (4.5): MP $212^{\circ} \mathrm{C}$; IR $\left(\mathrm{KBr}, \mathrm{cm}^{-1}\right): 3350,3125,17010,1525,1351 ;{ }^{1} \mathrm{H}$ NMR (DMSO-d $6,300 \mathrm{MHz}): 8.06(2 \mathrm{H}, \mathrm{d}, \mathrm{J}=7.2 \mathrm{~Hz}, \mathrm{Ar}), 7.76(2 \mathrm{H}, \mathrm{d}, \mathrm{J}=7.2 \mathrm{~Hz}, \mathrm{Ar}), 7.20(4 \mathrm{H}, \mathrm{m}, \mathrm{Ar})$, $3.57\left(1 \mathrm{H}, \mathrm{dd}, \mathrm{J}=10.8 \mathrm{~Hz}, \mathrm{CH}_{2}\right), 3.01\left(1 \mathrm{H}, \mathrm{dd}, \mathrm{J}=10.2 \mathrm{~Hz}, \mathrm{CH}_{2}\right), 4.80(1 \mathrm{H}, \mathrm{t}, \mathrm{J}=10.8 \mathrm{~Hz}, \mathrm{CH}), 4.60(1 \mathrm{H}, \mathrm{s}$, $\mathrm{NH}), 10.01\left(2 \mathrm{H}, \mathrm{s}, \mathrm{CONH}_{2}\right), 2.56\left(3 \mathrm{H}, \mathrm{s}, \mathrm{CH}_{3}\right) ;{ }^{13} \mathrm{C} \mathrm{NMR}$ (DMSO-d $\left.6,300 \mathrm{MHz}\right): 165.20,143.84,143.27$, 139.66, 138.08, 137.95, 134.63, 132.16, 128.79, 128.24, 127.69, 125.33, 124.14, 63.05, 42.45, 21.56, 10.47; MS: $m / \mathrm{z} 360.41\left[\mathrm{M}^{+}\right]$.

\section{4-(4-Methyl-5-(5-(p-Tolyl)-4,5-Dihydro-1H-Pyrazol-3-YI)-1H-1,2,3-Triazol-1-Yl)Benzamide}

(Colourless Amorphous Solid) (4.6): MP $212^{\circ} \mathrm{C}$; IR $\left(\mathrm{KBr}, \mathrm{cm}^{-1}\right): 3348,2911,1685,1500,1312 ;{ }^{1} \mathrm{H}$ NMR (DMSO-d $6,300 \mathrm{MHz}): 8.06(2 \mathrm{H}, \mathrm{d}, \mathrm{J}=8.4 \mathrm{~Hz}, \mathrm{Ar}), 7.76(2 \mathrm{H}, \mathrm{d}, \mathrm{J}=8.4 \mathrm{~Hz}, \mathrm{Ar}), 7.29(2 \mathrm{H}, \mathrm{d}$, $\mathrm{J}=7.8 \mathrm{~Hz}, \mathrm{Ar}), 717(2 \mathrm{H}, \mathrm{d}, \mathrm{J}=8.1 \mathrm{~Hz}, \mathrm{Ar}), 3.61\left(1 \mathrm{H}, \mathrm{dd}, \mathrm{J}=10.5 \mathrm{~Hz}, \mathrm{CH}_{2}\right), 3.00\left(1 \mathrm{H}, \mathrm{dd}, \mathrm{J}=10.2 \mathrm{~Hz}, \mathrm{CH}_{2}\right)$, $4.80(1 \mathrm{H}, \mathrm{t}, \mathrm{J}=3.0 \mathrm{~Hz}, \mathrm{CH}), 4.61(1 \mathrm{H}, \mathrm{s}, \mathrm{NH}), 10.0\left(1 \mathrm{H}, \mathrm{s}, \mathrm{NH}_{2}\right), 2.56\left(3 \mathrm{H}, \mathrm{s}, \mathrm{CH}_{3}\right), 2.29\left(3 \mathrm{H}, \mathrm{s}, \mathrm{CH}_{3}\right) ;{ }^{13} \mathrm{C}$ NMR (DMSO-d $6,300 \mathrm{MHz})$ : 165.20, 143.86, 140.30, 139.67, 138.08, 136.70, 134.63, 132.15, 129.43, 128.78, 126.96, 125.34, 62.84, 42.41, 21.16, 10.46; MS: $\mathrm{m} / \mathrm{z} 360.41\left[\mathrm{M}^{+}\right]$.

\section{4-(5-(5-(2-Hydroxyphenyl)-4,5-Dihydro-1 H-Pyrazol-3-Yl)-4-Methyl-1H-1,2,3-Triazol-1-}

Yl)Benzamide (Colourless Amorphous Solid) (4.7): MP 208 ${ }^{\circ}$; IR $\left(\mathrm{KBr}, \mathrm{cm}^{-1}\right): 3350,3009,1690$, 1550, 1351; ${ }^{1} \mathrm{H}$ NMR (DMSO-d $\left.6,400 \mathrm{MHz}\right): 8.06(2 \mathrm{H}, \mathrm{d}, \mathrm{J}=8.4 \mathrm{~Hz}, \mathrm{Ar}), 7.76(2 \mathrm{H}, \mathrm{d}, \mathrm{J}=8.4 \mathrm{~Hz}, \mathrm{Ar}), 7.29$ $(2 \mathrm{H}, \mathrm{d}, \mathrm{J}=7.5 \mathrm{~Hz}, \mathrm{Ar}), 6.85(2 \mathrm{H}, \mathrm{d}, \mathrm{J}=8.1 \mathrm{~Hz}, \mathrm{Ar}), 7.11(1 \mathrm{H}, \mathrm{t}, \mathrm{J}=7.5 \mathrm{~Hz}, \mathrm{Ar}), 6.80(1 \mathrm{H}, \mathrm{t}, \mathrm{J}=7.5 \mathrm{~Hz}, \mathrm{Ar})$, $3.56\left(1 \mathrm{H}, \mathrm{dd}, \mathrm{J}=10.2 \mathrm{~Hz} .10 .2 \mathrm{~Hz}, \mathrm{CH}_{2}\right), 2.87\left(1 \mathrm{H}, \mathrm{dd}, \mathrm{J}=9.9 \mathrm{~Hz}, 9.6 \mathrm{~Hz}, \mathrm{CH}_{2}\right), 5.03(1 \mathrm{H}, \mathrm{t}, \mathrm{J}=9.9 \mathrm{~Hz}, \mathrm{CH}$, $)$, $4.60(1 \mathrm{H}, \mathrm{s}, \mathrm{NH}), 10.00(1 \mathrm{H}, \mathrm{s}, \mathrm{OH}), 9.63\left(2 \mathrm{H}, \mathrm{s}, \mathrm{CONH}_{2}\right), 2.56\left(3 \mathrm{H}, \mathrm{s}, \mathrm{CH}_{3}\right) ;{ }^{13} \mathrm{C}$ NMR $\left(\mathrm{DMSO}_{6}, 300\right.$ MHz): 165.20, 143.84, 143.27, 139.66, 138.08, 137.95, 134.63, 132.16, 128.79, 128.24, 127.69, 125.33, 124.14, 63.05, 42.45, 21.56, 10.47, MS: $\mathrm{m} / \mathrm{z} 364.16\left[\mathrm{M}^{+}\right]$.

4-(5-(5-(4-Fluorophenyl)-4,5-Dihydro-1H-Pyrazol-3-Yl)-4-Methyl-1H-1,2,3-Triazol-1-Yl)Benzamide (Colourless Amorphous Solid) (4.8): MP $219^{\circ} \mathrm{C}$; IR $\left(\mathrm{KBr}, \mathrm{cm}^{-1}\right): 3352,2910,1610,1510,1310 ;{ }^{1} \mathrm{H}$ NMR (DMSO-d $6,300 \mathrm{MHz}): 8.06(2 \mathrm{H}, \mathrm{d}, \mathrm{Ar}-\mathrm{H}, \mathrm{J}=7.5 \mathrm{~Hz}), 7.22(2 \mathrm{H}, \mathrm{d}, \mathrm{Ar}-\mathrm{H}, \mathrm{J}=8.1 \mathrm{~Hz}), 7.76(2 \mathrm{H}, \mathrm{d}$, $\mathrm{J}=8.1 \mathrm{~Hz}, \mathrm{Ar}), 7.74(2 \mathrm{H}, \mathrm{d}, \mathrm{J}=9.6 \mathrm{~Hz}, \mathrm{Ar}), 3.65\left(1 \mathrm{H}, \mathrm{dd}, \mathrm{J}=11.4 \mathrm{~Hz}, \mathrm{CH}_{2}\right), 3.01\left(1 \mathrm{H}, \mathrm{dd}, \mathrm{J}=10.2 \mathrm{~Hz}, \mathrm{CH}_{2}\right)$, $4.88(1 \mathrm{H}, \mathrm{t}, \mathrm{J}=9.3 \mathrm{~Hz}, \mathrm{CH}), 10.01\left(2 \mathrm{H}, \mathrm{s}, \mathrm{CONH}_{2}\right), 4.60(1 \mathrm{H}, \mathrm{s}, \mathrm{NH}), 2.56\left(3 \mathrm{H}, \mathrm{s}, \mathrm{CH}_{3}\right) ;{ }^{13} \mathrm{C} \mathrm{NMR}$ (DMSO-d $\left.{ }_{6}, 300 \mathrm{MHz}\right): 165.21,163.44,160.23,143.99,139.58,139.43,138.06,134.65,132.24,129.08$, $128.98,128.79,125.35,115.75,115.47,62.37,42.45,10.46$; MS: $\mathrm{m} / \mathrm{z} 364.38\left[\mathrm{M}^{+}\right]$.

\section{4-(5-(5-(4-Bromophenyl)-4,5-Dihydro-1H-Pyrazol-3-Yl)-4-Methyl-1H-1,2,3-Triazol-1-}

Yl)Benzamide (Pale Yellow Color Amorphous Solid) (4.9): MP $241^{\circ} \mathrm{C}$. IR $\left(\mathrm{KBr}, \mathrm{cm}^{-1}\right): 3345,2900$, 1620, 1551, 1320,; ${ }^{1} \mathrm{H}$ NMR (DMSO-d $6,300 \mathrm{MHz}$ ): 8.06 (2H, d, J=8.4Hz, Ar), 7.57 (2H, d, J=8.4Hz, Ar), $7.76(2 \mathrm{H}, \mathrm{d}, \mathrm{J}=8.7 \mathrm{~Hz}, \mathrm{Ar}), 7.38(2 \mathrm{H}, \mathrm{d}, \mathrm{J}=8.4 \mathrm{~Hz}, \mathrm{Ar}), 3.66\left(1 \mathrm{H}, \mathrm{dd}, \mathrm{J}=10.8 \mathrm{~Hz}, 10.5 \mathrm{~Hz}, \mathrm{CH}_{2}\right), 3.0(1 \mathrm{H}$, $\left.\mathrm{dd}, \mathrm{J}=10.2 \mathrm{~Hz}, 9.9 \mathrm{~Hz}, \mathrm{CH}_{2}\right), 4.84(1 \mathrm{H}, \mathrm{t}, \mathrm{J}=3.0 \mathrm{~Hz} \mathrm{CH}), 10.01\left(2 \mathrm{H}, \mathrm{s}, \mathrm{CONH}_{2}\right), 4.64(1 \mathrm{H}, \mathrm{s}, \mathrm{NH}), 2.56$ $\left(3 \mathrm{H}, \mathrm{s}, \mathrm{CH}_{3}\right) ;{ }^{13} \mathrm{C}$ NMR (DMSO-d 6 , $300 \mathrm{MHz}$ ): 165.20, 143.98, 142.82, 139.52 138.05, 134.65, 132.27, $131.77,129.37,128.79,125.34,120.61,62.36,42.37,10.47$; MS: $\mathrm{m} / \mathrm{z} 426.1\left[\mathrm{M}^{+}\right] 428.1[\mathrm{M}+2]$.

\section{RESULTS AND DISCUSSION}

In our previous work, a series of $p$ - aminobenzamide derived chalcones were synthesized and evaluated for antibacterial, antifungal, and antiproliferation activities and compared to the standard drugs ${ }^{5}$. Diverse applications of 1,2,3 - triazole and pyrazolines prompted us to synthesize 1,2,3 - triazole linked pyrazolines and to evaluate their biological activity. Following the basic chemical reactions published in the literature 4- amino benzamide was converted into a 1,2,3-triazole through the corresponding azide. The synthesized triazole bearing acyl group at position 5 facilitates the preparation of chalcones. In the 
RASĀYAN J. Chem.

Vol. 12 | No. 4 |2260 - 2266| October - December | 2019

present work, we attempted to synthesize a series of pyrazolines using condensation reaction between the chalcones and hydrazine hydrate in acetic acid medium. The conversion of 4- aminobenzamide into azide 1 was confirmed by $\mathrm{N}=\mathrm{N}$ stretching absorption band at around $1500 \mathrm{~cm}^{-1}$ in its IR spectrum and devoid of $\mathrm{N}-\mathrm{H}$ stretching frequency at $3450 \mathrm{~cm}^{-1}$ of amino group in the starting material and two doublets arrived at $\delta$ value 7.20 and 7.93 revealed that azide would be a $p$ - disubstituted compound. The formation of 4-(5-acetyl-4-methyl-1H-1, 2, 3-triazole-1-yl) benzamide 2 was ensured from a three proton singlet that appeared at $\delta$ value 2.55 and 2.64 for methyl and acyl protons respectively. The 5- acetyltriazole was subjected to a condensation reaction with substituted benzaldehyde to prepare a series of chalcones 3.1- 3.9. The formation of chalcone was confirmed from two doublets integrated for one proton each arrived between $\delta 7.94$ and 8.21 for $\mathrm{H} \alpha$ and $\mathrm{H} \beta$ protons respectively. A series of target compounds pyrazolines 4.1- 4.9 were synthesized from a reaction between chalcones and hydrazine hydrate in the presence of an acid medium. The formation of pyrazolines was confirmed from two double doublets between $\delta 2.8 \& \delta 3.5$ and a triplet between $\delta 4.8 \& \delta 5.0$ corresponding to stereo chemically nonequivalent protons of pyrazoline ring. Appreciable antimicrobial activity compared to standard was observed from halogen-substituted chalcones and pyrazolines in particular bromine and fluorine substituted chalcones and pyrazolines.

\section{CONCLUSION}

4-amino benzamide derived 1, 2, 3 triazole linked pyrazolines were synthesized through their chalcone intermediate in appreciable yield and evaluated for their antimicrobial activity to identify the potential molecule. In our previous work 4-aminobenzamide was converted into its 1,2,3-triazole linked chalcones and evaluated for their antimicrobial and antiproliferative studies. All the chalcones have shown good anti bacterial activity compared to the standard drug streptomycin. It was concluded that halogen-substituted chalcones and pyrazoline have shown appreciable activity compared to standard drugs.

The influence of extended of conjugation and electro negativity of the functional groups on the aldehydes moiety was assumed to be responsible for the observed biological activity of the synthesized compounds. But compared to the amido group even then the nitro group possesses higher electro negativity and also greater extent of conjugation is not reflected on the microbial activity observed. So that incorporation of structural modification in the 1,2,3-triazole-chalcone hybrid by incorporating the pyrazoline ring may not be a suitable chemical environment to achieve better antimicrobial compounds compared to their parent compounds 1,2,3-triazole-chalcone hybrid was concluded.

\section{ACKNOWLEDGMENT}

The authors thank the management of Vivekanandha Educational Institutions for providing laboratory facilities, SASTRA University and IIT Madras for providing spectral data.

\section{REFERENCES}

1. SH. Shah, S. Pankaj, PS. Patel, M. Prajapati, International Journal of Pharmaceutical Sciences and Research, 14(1), 4113(2014), DOI:10.13040/IJPSR.0975-8232.6(10) 4113-2

2. M. E. Welsch, S. A. Snyder, B. R. Stockwell, Current Opinion in Chemical Biology, 14(3), 347(2010), DOI:10.1016/j.cbpa.2010.02.018

3. K. S. S. Praveena, N.Y.S.Murthy, S.Pal, Journal of Chemical and Pharmaceutical Research, 7, 506(2015).

4. O. P. Pravin, B. B. Sanjay, Arabian Journal of Chemistry, 9(4), 588(2016), DOI: 10.1016/j.arabjc.2013.08.027

5. N. Hari, S. Aravind, G. Kurian, R. Rajmohan, R. Ravikumar, Der Pharmacia Lettre, 8, 275(2016).

6. B. Zhou, C. Xing, Medicinal Chemistry, 5, 388(2015), DOI:10.4172/2161-0444.1000291

7. D. I. Batovska, I. T. Todorova, Current Clinical Pharmacology, 5, 1(2010), DOI: 10.2174/157488410790410579.

8. N. K. Sahu, S. S. Balbhadra, J. Choudhary, D. V. Kohli, Curr. Med. Chem., 19, 209(2012), DOI: 10.1016/S1872-2067(14)60247-3,

9. P. Singh, A. Anand, V. Kumar, European Journal of Medicinal Chemistry, 85, 758(2014), DOI :10.1016/j.ejmech.2014.08.033 
RASĀYAN J. Chem.

Vol. 12 | No. 4 |2260 - 2266| October - December | 2019

10. C. Karthikeyan, N. S. Mootrhy, S.Ramasamy, U. Vanum, E. Manivannan, D. Karunagaran, P. Trivedi, Recent Patents on Anti-Cancer Drug Discovery, 10, 97(2015), DOI: $10.2174 / 1574892809666140819153902$

11. S. Viveka, Dinesha, P. Shama, G. K. Nagaraja, S. Ballav, S. Kerkar, European Journal of Medicinal Chemistry, 101, 442 (2015), DOI: 10.4155/fmc-2017-0144

12. J. M. Pandya, Mahyavanshi, Rasayan Journal of Chemistry, 12(3), 1229 (2019), DOI: 10.31788/RJC.2019.1235033

13. M. Toshniwal, M. Bundel, P. Kriplani, R. Pareek, A. Pareek, Rasayan Journal of Chemistry, 12(3), 1294 (2019), DOI:10.31788/RJC.2019.1235207

14. P. Chaitanya, G. Ravi, S. Damodhar, A. Ravindernath, Rasayan Journal of Chemistry, 11(3), 1357 (2018), DOI: 10.31788/RJC.2018.1131599

15. M. Idrees, S. Kola, N. J. siddiqui, Rasayan Journal of Chemistry, 12(4), 1725(2019), DOI: 10.31788/RJC.2019.1245467

16. A. Adhikari, B. Kalluraya, K. V. Sujith, K. Gouthamchandra, R. Jairam, R. Mahmood, R. Sankolli, European Journal of Medicinal Chemistry, 55, 467(2012), DOI:10.13040/IJPSR.09758232.6(10).4113-28

17. R. Aggarwal, S. Kumar, P. Kaushik, D. Kaushik, G. K. Gupta, European Journal of Medicinal Chemistry, 62, 508 (2013), DOI:10.1016/j.ejmech.2012.11.046

18. B. N. Acharya, D. Saraswat, M. Tiwari, A. K. Shrivastava, R. Ghorpade, S. Bapna, M. P. Kaushik, European Journal of Medicinal Chemistry, 45, 430 (2010), DOI: 10.1016/j.ejmech.2009.10.023.

19. A. Adhikari, B. Kalluraya, K. V. Sujith, K. Gouthamchandra, R. Jairam, R. Mahmood, R. Sankolli, European Journal of Medicinal Chemistry, 55, 467 (2012), DOI:10.1016/j.ejmech.2012.07.002

20. E. A. Begum, Y. C. Samiye, U. Gulberg, Y. Kemal, E. Rahmiye, Bioorganic \&. Medicinal Chemistry. Letters, 24, 3278 (2014), DOI: 10.1016/j.bmcl.2014.06.015

21. T. Xu, C. Rui, Z. Tong-Tian, H. Yan, T. Wen-Jian, L. Xin-Hua, Bioorganic \&. Medicinal Chemistry Letters, 23, 515 (2015), DOI: 10.1016/j.bmc.2014.12.010

22. C. K. Sharad, B. P. Vishal, K. R. Dipak, European Journal of Medicinal Chemistry, 84, 51(2014), DOI: $10.1016 /$ j.ejmech.2014.07.008

23. J. A. Mohamed, G. S. Jeyabalan, S. Savita, J. Naresh, K. Lalit, K. S. Lalit, Y. Hemant, S. Lokesh, G. K. Rajput, S. D. Narendra, P. Ravindra, B. J. Chandra, Bioorganic \&. Medicinal Chemistry Letters, 21, 5259(2011), DOI:10.1016/j.bmcl.2011.07.035

24. F. A. Bakr, A. Ehab, A. M. Hanan, E. A. A. Ghada, European Journal of Medicinal Chemistry, 52, 263 (2012), DOI:10.1016/j.ejmech.2012.03.023

25. Y. W. Mohmmad, R. B. Abdul, A. Amir, H. L. Dae, C. Inho, A. Fareeda, European Journal of Medicinal Chemistry,54, 845 (2012), DOI:10.1016/j.ejmech.2012.03.049

26. H. Khalilullah, S. Khan, M. J. Ahsan, B. Ahmed, Bioorganic \&. Medicinal Chemistry Letters, 21, 7251, (2011), DOI:10.1016/j.bmcl.2011.10.056

27. M. R. Bhosle, A. R. Deshmukh, S. Pal, A. K. Srivastava, R. A. Mane, Bioorganic \&. Medicinal Chemistry Letters, 25, 2442, (2015), DOI:10.1016/j.bmcl.2015.03.068

28. R. Fioravanti, A. Bolasco, F. Manna, F. Rossi, F. Orallo, F. Ortuso, S. Alcaro, R. Cirilli, European Journal of Medicinal Chemistry, 45, 6135, 2010, DOI: 10.1016/j.ejmech.2010.10.005

29. C. P. Kaushik, R. Luxmi, D. Singh, A. Kumar, Molecular Diversity, 21, 137(2017), DOI: 10.1007/s11030-016-9710-y.

30. V. R. Kamalraj, S. Senthil, P. Kannan, Journal of Molecular Structure, 892, 210 (2008), DOI: 10.1016/j.molstruc.2008.05.028

31. N. T. Pokhodylo, R. D. Savka, V. S. Matiichuk, N. D. Obushak, Russian Journal of General Chemistry, 79, 389 (2009) DOI:10.1134/S1070363209020248

32. H. S. Dong, H. C. Wang, Z. L. Gao, R. S. Li, F. H. Cui, J. Heterocycl. Chem., 47, 389, (2010), DOI: $10.1002 /$ jhet.336

[RJC-5383/2019] 